\title{
Linear Programming with Uncertain Data : Some Extensions to Robust Optimization
}

\author{
B D Craven,* and S M N Islam ${ }^{\dagger}$
}

March 20, 2012

\begin{abstract}
An optimization problem often has some uncertain data, and the optimum of a linear program can be very sensitive to small changes in the data. Such a problem can often be modified to a robust program, which is more stable to such changes. Various methods for this are compared, including requiring all versions of the data to be satisfied together (but they may be inconsistent), worst-case MAX-MIN model, and various models where deviations incur penalty costs. Existing methods require substantial computation. It is shown here that smaller computations often suffice; not all cases need be considered. Other penalty methods are suggested, using different norms. Moreover, perturbations of constraint coefficients can be represented by suitable perturbations of a requirement vector.
\end{abstract}

KeyWords linear proramming, robust optimization, uncertain data.

AMS Classification $\quad 90 \mathrm{C} 05$

${ }^{*}$ Dr B D Craven, Dept. of Mathematics \& Statistics, University of Melbourne,Victoria 3010, Australia brucedc37@gmail.com (Corresponding author)

${ }^{\dagger}$ Prof. S M N Islam, Victoria University, PO Box 14428, Melbourne, Vic. 8001, Australia sardar.islam@vu.edu.au. 


\section{Introduction}

The data set of a linear program (consisting of a matrix of coefficients and a requirements vector) is ill-conditioned (see Section 3) if a small change in some elements of the data set makes a large change in the optimal vector. The optimal objective value will sometimes not be changed very much. In such situations, the linear program may need to be reformulated to give a good description of a real problem.

Some authors have discussed robust optimization, meaning that the solution should be a fixed vector $x$, that remains feasible for every perturbed version of the constraints, provided that the perturbations lie in prescribed regions. This "naive" approach (so described by Greenberg and Morrison [1] .

This tacitly assumes that the constraints are hard (must be satisfied exactly), but some of the data is not known precisely.

Instead, one may consider a "worst-case" formulation, leading to a MAX-MIN problem (which may, however, raise computational issues). When the given linear program is described by equality constraints, small changes in the data set can also be described by changes in the requirements of the program (see Section 8).

The small changes in the dataset, describing different versions of the linear program, represent uncertainty in the problem, though not necessarily stochastic elements. The uncertainty will be subject to some bounds. A stochastic model is appropriate in circumstances when the system will be run a number of times, with different versions of data obtained by independent samplings from some probability distribution.

However, the reformulated problem might still be ill-conditioned, with the optimum not precisely determined computationally by the model. In such a situation, a soft constraint might also be considered, with a penalty cost when it is not precisely satisfied.

This paper proposes several alternative methods for robust optimization, which are easy to formulate, and require less computation. Section 2 briefly presents the existing methods for formulating and computing robust versions of linear programs with uncertain data. Section 3 discusses ill-conditioning, and approximation of feasible region by fewer constraints. Section 4 presents the variant forms of linear program that need 
consideration. Section 5 develops a robust model for equality constraints,and discusses how the resulting MAX-MIN model could be computed. Section 6 discusses a robust model for inequality constraints, and shows how to minimize the computation, when many versions of constraints are involved. Section 7 describes the use of penalty functions, when the constraints cannot be satisfied exactly. Section 8 shows that perturbed inequality constraints can be represented as perturbations of the requirements vector. Section 9 describes several other variants of penalty methods. Section 10 presents the conclusions.

\section{Literature Review}

Some authors, including Ben-Tal et al. [2] and Greenberg and Morrison [1] have presented robust modifications of linear programs, which require all versions of the constraints to be satisfied together. But that construction leads to inconsistencies for many linear programs. Alternative formulations are worst case models, for which see Kouvelis and Gang [3], Mulvey and Vanderbei [4], Ruszczyński and Shapiro [5]. leading to MIN-MAX (or MAX-MIN) problems (which may present computational questions), and also several penalty approaches, including those of Tapiero [6] and Dupačová and Stepan [7].

\section{Ill-Conditioned}

A linear program has (except in special cases) a unique optimum at a vertex of the feasible set. But the optimum point is not always computationally well defined. If the constraints are $A x=b, x \geq 0$, with a $m \times n$ matrix $A$, then the nonzero elements of the optimal $x$ are included in the vector $M^{-1} b$, where $M$ is an appropriate $m \times m$ submatrix of $A$. But $M$ may be ill-conditioned, and then a small change in $b$ may produce a large change in $x$. Since computation is necessarily to a finite precision, ill-conditioning may lead to a computed optimum that is seriously in error.

The condition number $\kappa(M)$ of $M$ is the maximum ratio of the relative error in the solution $x$ divided by the relative error in $b$. Then $\kappa(M)=\|M\| \cdot\left\|M^{-1}\right\|$ is given by 
the ratio of the maximal to minimal singular values of $M$.

If $M$ is perturbed to $M+P$, where $\|P\|$ is small, then

$$
(M+P)^{-1} \approx M^{-1}-M^{-1} P M^{-1},
$$

so that a large condition number for $M$ leads to sensitivity of the solution to small changes in $M$.

Hence a small value of $\operatorname{MAX}_{M} \kappa(M)$ (were it practicable to compute it) would indicate stability of the linear program to small changes in the data.

\section{Formulations}

Consider a linear program, containing a parameter $q$, in either of the forms

$$
\begin{aligned}
& \operatorname{MIN} x c^{T} x \text { subject to } x \geq 0, A(q) x=b(q) ; \\
& \text { MIN } x c^{T} x \text { subject to } x \geq 0, A(q) x \leq b(q) .
\end{aligned}
$$

Here the elements of $A$ and $b$ depend on a parameter $q$, required to lie in a region $U$. Thus different values of $q$ lead to different versions of the objective and constraints. If $c$ also depends on $q$, then $\operatorname{MIN}_{x} c(q)^{T} x \ldots$ can be replaced by

$$
\operatorname{MIN}_{x, t} t \text { subject to } c(q)^{T} x \leq t, \ldots
$$

One approach to robustness for (LP2) is to include all the versions of the constraints in a single (larger) linear program, so that all versions of the constraints must be feasible together. For example, Ben-Tal et al. [2] consider

$$
\mathrm{M} \mathrm{IN} \sup _{c, A, T} c^{T} x \text { subject to } A x \leq b(\forall(c, A, b) \in U)
$$

expressed as

$$
\operatorname{MIN}_{x, t} t \text { subject to } c^{T} x \leq t, A x \leq b \quad(\forall(c, A, b) \in U)
$$

However, this approach does not work for (LP1), since the equality constraints will be inconsistent if there are more than a few points in $U$, or values of the parameter $q$. While (LP1) and (LP2) are computationally equivalent, the conversion from (LP1) to (LP2) requires artificial variables, and so changes the model, and its stability properties. 


\section{Equality Constraints; Computational Methods}

Consider the robust model:

$\operatorname{MAX}_{q \in U} \varphi(q)$ where $\varphi(q):=:=\operatorname{MIN}_{x} c^{T} x$ subject to $x \geq 0, A(q) x=b(q)$.

Here the linear program includes only one version of the constraint, and the worst choice of that version is sought. (See Kouvelis and Gang 2010, Mulvey and Vanderbei 1995, Ruszczyński and Shapiro 2000 for MAX-MIN or MIN MAX formulations. But more consideration is needed of computational approaches.)

The inner problem has Lagrangian

$$
L(x, \lambda, \theta, q): c^{T} x+\lambda^{T}(A(q) x-b(q))-\theta^{T} x
$$

and the gradient

$$
\varphi^{\prime}(q)=\frac{\partial}{\partial q} L(x, \lambda, \theta, q)=\lambda\left(A^{\prime}(q) x-b^{\prime}(q)\right),
$$

where prime denotes gradient, and $x$ takes its optimal value.

Suppose now that $U$ is a box, specified by constraints $-\alpha_{s} \leq q_{s} \leq \beta_{s}$. The outer problem may be computed by a penalty method:

$$
\operatorname{MAX}_{q} \varphi(q)-\frac{1}{2} \mu \sum_{s}\left\{\left[q_{s}-\beta-\epsilon_{s}\right]_{+}^{2}+\left[-q_{s}-\alpha-\epsilon_{s}^{\prime}\right]_{+}^{2}\right\}
$$

where $[z]_{+}=z$ if $z>0$, otherwise $0, \mu$ is a positive weight, and suitable $\epsilon>0$ makes the constraints exactly satisfied. Here $\epsilon_{s}$ and $\epsilon_{s}^{\prime}$ depend on Lagrange multipliers, which need not be computed unless $U$ is a precisely specified region. However, $\varphi(\cdot)$ is not a concave function, so computing its maximum may present problems.

\section{Inequality Constraints; Computational Methods}

Consider the constraint $A(q) x \leq b(q)$ of (LP2) in Section 4 , where $A(\cdot)$ and $b(\cdot)$ are affine functions (constant + linear), and $q \in U$, a polyhedron with vertices $s_{1}, s_{2}, \ldots$ Denote by $V$ the set of these vertices. Since $A(\cdot)$ and $b(\cdot)$ are affine,

$$
(\forall q \in V) A(q) x \leq b(q) \Leftrightarrow \forall q \in U) A(q) x \leq b(q)
$$


Hence the problem:

$$
\operatorname{MIN} x c^{T} x \text { subject to } x \geq 0, A(q) x=b(q)(\forall q \in V)
$$

is equivalent to the similar problem (LP2) with $(\forall q \in U)$.

Here $U$ could be a box, as in Section 5. Alternatively (with less computation), choose $V=\left\{v_{0}, v_{1}, v_{2}, \ldots, v_{r}\right\}$, with the $b_{j}$ constructed sequentially with

$$
v_{j+1} \notin \operatorname{span}\left\{v_{0}, \ldots, v_{j}\right\},
$$

so that $0 \in \operatorname{int} U$, and $U$ has dimension $r$. Otherwise, if $U$ is bounded convex, then $(\exists k)(\forall q \in U) q \geq-k$, and $S:=U+k$ may be approximated by

$$
(\forall s \in S) s \geq 0, e^{T} s \leq 0
$$

for $e=(1,1, \ldots)$.

(For other results with inequality constraints, see Greenberg and Morrison 2008 and Ben-Tal, El Ghaoui and Nemirovski 2009.)

\section{Penalty Approach}

Since usually the same $x$ does not satisfy $A(q) x=b(q)$ for all $q \in U$, consider the modified constraint:

$$
(\forall q \in U) A(q) x=b(q)+u(x, q),
$$

with a "small" compensation term $u(x, q)$, and a minimizing objective:

$$
c^{T} x+\gamma\|u(x, \cdot)\|
$$

with some suitable norm and weight $\gamma$, in particular with $\|u(x, \cdot)\|=\operatorname{MAX}_{q}|w(q) u(x, q)|$.

This penalty approach also applies to soft constraints, which need not be satisfied exactly.

Section 9 describes some variants of the penalty approach. 


\section{Inequality Constraints as Perturbations}

Consider (LP2) with the constraints rewritten as

$$
(\bar{A}+Q) x \leq \bar{b}+g, x \geq 0,
$$

where $\bar{A}$ and $\bar{b}$ do not depend on $q, Q$ is a sparse matrix some of whose components depend on $q$, and $g$ depends on $q$. Assume $\bar{x}$ is optimal when $Q=0$ and $g=0$. Then:

$$
\bar{A} x \leq \bar{b}+g-Q x,
$$

with the changes in $A$ appearing as perturbations of the requirement $\bar{b}$, but the term $-Q x$ still to be determined. Suppose that $Q$ and $g$ are constrained by $Q \geq-M$ and $g \leq \gamma$, with componentwise inequalities, and constant matrix $M$ and vector $\gamma$. With $x \geq 0, Q \geq-M \Rightarrow-Q x \leq M x$. A similar inequality holds for $g \leq \gamma$. So the constraint

$$
\bar{A} x \leq \bar{b}+\gamma+M x
$$

implies the constraint with $-Q$ x replacing $M x$, for each $Q \geq-M$, and $\gamma$ replacing $g$ for each $g \leq \gamma$.

Consider then a seqence $x^{t}$ generated by

$$
x^{t+1}=\arg \max c^{T} x \text { subject to } \bar{A} x=\bar{b}+\gamma+M x^{t} \quad(t=0,1, \ldots) ; \quad x^{0}=\bar{x} .
$$

Since $x^{t+1}=B^{-1}\left(\bar{b}+\gamma+M x^{t}\right)$, where $B$ is some basis matrix from $\bar{A}$, and the set of $B^{-1}$ ar norm-bounded, it follows that the mapping $x^{t} \rightarrow x^{t+1}$ is a contraction mapping, provided that $\gamma$ and $M$ have sufficiently small norms; then the sequence $\left\{x^{t}\right.$ converges, say to $\xi$. Then the robust model is solved by the linear program:

$$
\operatorname{MIN}_{x} c^{T} x \text { subject to } \bar{A} x \leq \bar{b}+\gamma+M \xi
$$

It is remarked that this robust model for (LP2) is applicable provided that $\|\xi-\bar{x}\|$ is not too large; otherwise the linear program is too unstable to be useful.

\section{Vector Models}

For $q \in U$, let $z(q):=\operatorname{MIN}_{x}\left\{c(q)^{T} x: x \geq 0, A(q) x=b(q)\right.$; denote by $z$ the vector of $z(q)$ for $q \in U$, by $\psi(x)$ the vector of $z(q)-c(q)^{T} x$ for $q \in U$, and by $\theta(x)$ the vector 
of $A(q) x-b(q)$ for $q \in U$. Both these norms cannot be zero. However, the further problem:

$$
\operatorname{MIN}_{x}\|\psi(x)\|+\gamma\|\theta(x)\|,
$$

for some norms and some weight $\gamma$, seeks to minimize them. Suitable norms are $\|s\|:+\operatorname{MAX}_{q}|s(q)|$ or $\sum w(q)|s(q)|$, with weights $w(q)$; these correspond to regret (see Kouvelis and Gang 2010) and tracking models. (Tapiero 1998 introduces a variance term. It would be dimensionally more appropriate to use the square root, thus a $\ell^{2}$ norm.)

Another version of tracking (see Dupačová and Stepan 2002) is the following. For $q \in U$, denote

$$
v(q):=\operatorname{MIN}\left\{c^{T} x: \bar{A} x=\bar{b}, A^{q} x=b^{q}, x \geq 0,\right.
$$

where $\bar{A}$ and $\bar{b}$ are fixed, and $A^{q}$ and $b^{q}$ are uncertain, depending on the parameter $q \in U$. With weights $p^{q}$, summing to 1 , (which may, but need not, have a probability interpretation) and a weight $\gamma$, the second minimization:

$$
\operatorname{MIN}_{x} \sum_{q \in U} p^{q}\left[\left\|c^{T} x-v(q)\right\|+\gamma\left\|A^{q} x-b^{q}\right\|\right]
$$

compromises between minimizing the objective and satisfying the uncertain constraints.

\section{Conclusions}

The optimum point of a linear program with some uncertain (perhaps random) data may be very sensitive to small changes in some data. Various methods for stabilizing such a program are compared, and some improved modelling and computing methods proposed. Some have called robust a model where all versions of the constraints must hold together; but this can only apply in restricted circumstances. More general alternatives include worst case, and a class of penalty methods. A model with some uncertain coefficients can be related to a model with an uncertain requirements vector, to which the shadow price concept may apply. This paper has just developed some alternative approaches to robust optimization, which are less restrictive and require less computation. 


\section{References}

1. Greenberg H J and Morrison T 2008. Robust Opimization, Chapter 14 of Ravindran A and Ravi A, Operations Research Methodologies, CRC, Hoboken.

2. Ben-Tal A, El Ghaoui L and Nemirovski A, 2009. Robust Optimization. Princeton University Press, Princeton.

3. Kouvelis P and Yu Gang 2010. Robust Discrete Optimization and its Applications, Kluwer, Dordrecht.

4. Mulvey J M and Vanderbei R J 1995. Robust Optimization of Large-Scale Systems, Operations Research 41(2), 264-281.

5. Ruczczyński and Shapiro (eds.) 2000. Stochastic Programming, Elsevier.

6. Tapiero C S 1998. Applied Stochastic Models and Control for Finance and Insurance, Kluwer, Dordrecht.

7. Dupačová and Stepan J 2002. Stochastic Modeling in Economics and Finance. Kluwer, Dordrecht. 


\section{University Library}

\section{- M M N E R VA A gateway to Melbourne's research publications}

Minerva Access is the Institutional Repository of The University of Melbourne

Author/s:

Craven, BD;Islam, SMN

Title:

Linear Programming with Uncertain Data: Some Extensions to Robust Optimization

Date:

2012-11-01

Citation:

Craven, B. D. \& Islam, S. M. N. (2012). Linear Programming with Uncertain Data:

Some Extensions to Robust Optimization. JOURNAL OF OPTIMIZATION THEORY AND APPLICATIONS, 155 (2), pp.673-679. https://doi.org/10.1007/s10957-012-0035-4.

Persistent Link:

http://hdl.handle.net/11343/282928 